\title{
Diversity of butterflies in a tropical rain forest of Van Ban Nature Reserve, Lao Cai Province, Vietnam (Lepidoptera: Rhopalocera)
}

\author{
Разнообразие дневных бабочек в дождевом тропическом месу \\ заповедника Ван-Бан (провинџия Мао-Кай, Вьетнам) \\ (Lepidoptera: Rhopalocera)
}

\author{
Vu Van Lien

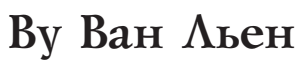

Vietnam National Museum of Nature, 18 Hoang Quoc Viet, Cau Giay, Hanoi, Vietnam. Email: vulien@gmail.com Вьетнамский Национальный музей природы, Ханой, Вьетнам

KEY WORDS. Butterfly, abundance, species, diversity, altitude, forest, stream side, grassy area. КЛЮЧЕВЫЕ СЛОВА. Бабочка, обилие, вид, разнообразие, высота, лес, берег реки, луг.

ABSTRACT. Diversity of butterfly communities of a tropical rain forest of Van Ban Nature Reserve in North Vietnam was studied in different habitat types (natural forests, stream sides in the forest, and grassy areas) at low (400-500 $\mathrm{m}$ a.s.1.) and high altitudes (800900 m a.s.1.) in March and April 2005. A total of 1,115 individuals from 170 butterfly species were recorded. The stream sides have the greatest, while the grassy areas the lowest, species and individual numbers. Low elevations yield more species and individuals than higher ones. Similarity of the species composition of butterfly communities is greater between similar habitats (natural forests at low and high altitudes, stream sides at low and high altitudes, and grassy areas at low and high altitudes). The habitat appears to be the main factor to affect the similarity of species composition between areas. Stream sides in forests play an important role in conserving the high species richness and the abundance of butterfly communities.

РЕЗЮМЕ. Разнообразие сообществ дневных бабочек дождевого тропического леса в заповеднике Ван-Бан в Северном Вьетнаме изучали в разных типах биотопов (естественный лес, берег реки и луг) низко (400-500 м н.у.м.) и высоко в горах (800-900 м н.у.м.) в марте-апреле 2005 г. Всего собрано 1115 экземпляров бабочек 170 видов. Наибольшее разнообразие видов и обилие отмечены по берегам рек, а самые низкие - на лугах. Для низкогорий отмечен максимум как видового, так и численного обилия. Сходство видового состава сообществ бабочек выше в однотипных биотопах (естественные леса, берега рек, а также луга в низкогрье и высокогорье, соответственно). Биотоп оказывается главным фактором, влияющим на сходство видового состава между территориями. Берега рек в лесу играют важную роль в сохранении большого видового богатства и обилия сообществ бабочек.

\section{Introduction}

Tropical forests are long known to contain great biodiversity levels, with insects playing important roles in forest ecosystems. Butterflies as well as other insects are distributed differently in different habitat types. Their diversity mainly depends on

vegetation. In general, insect diversity is the highest in habitats with the highest plant diversity, being the lowest in grassy and open areas [DeVries, 1992]. Different insect groups show different diversity trends. Some groups such as beetles and moths have high diversity levels in natural forests, but lower ones in secondary forests [Morse et al., 1988; Barlow \& Woiwod, 1989]. In contrast, butterflies tend to show low diversity levels in natural forests, but higher ones in disturbed forests [Brown, 1991; Blair \& Launer, 1997; Schulze et al., 2004; Fermon et al., 2005; Bobo et al., 2006]. Further studies yield that the numbers of butterfly species and individuals are higher in secondary and regenerating forests, again lower in natural forests [Spitzer et al., 1993; Vu \& Yuan, 2003], being the highest at forest edges and the lowest in agricultural lands [Vu, 2009].

There are plenty of streams in tropical forest. Open habitats in forest support more butterfly species than the forest canopy does [Hill et al., 2001]. Stream sides with open spaces may have more butterfly species and individuals than the natural closed forest, because many butterflies visit and take water and nutrients from sand and rocks along stream sides in forests.

The diversity of butterfly communities has been studied in different habitat types in different parts of the world. However, there have been few studies on the diversity of butterfly communities along stream sides in tropical forest. These habitats may play important roles in conserving a portion of tropical biodiversity, of which insects are a major part. Yet little data are available. 
Forest edges with more exposure to the open also show the greatest diversity of butterflies [Vu, 2009]. Gaps in forest sustain higher diversity levels of butterflies than closed forest areas do [Spitzer et al., 1997]. Stream sides with more open spaces could therefore be expected to support higher diversity levels of butterflies.

The present study suggests that stream sides in tropical forest may have more butterflies, both their abundance and species diversity, than natural forests and grassy areas do. Both shrub and grassy habitats are already known to maintain higher levels of butterfly diversity than both natural forest and agricultural lands [Vu, 2009]. But what is the situation concerning the diversity of butterfly communities in grassy areas alone? Our hypothesis is that grassy habitats support a lower level of butterfly diversity than the natural forest does.

\section{Materials and methods}

\section{Research site}

Research was carried out in Nam Xay commune of the Van Ban Nature Reserve, Van Ban District, Lao Cai Province, Vietnam $\left(21^{\circ} 58^{\prime} \mathrm{N}, 104^{\circ} 02^{\prime} \mathrm{E}\right)$ from March 15 to April 14, 2005. The study area is located on a mountain, with elevations ranging from 400 to more than $2,000 \mathrm{~m}$ a.s.1.

Six different 500 -m transects were set up in different habitat types and at two different altitudes, covering natural closed forests, stream sides in the forest, and grassy areas, one each at low altitudes of 400-500 m and at high altitudes at $800-900 \mathrm{~m}$ a.s.l. The habitats are as following:

Natural forests at the lower (NFl) and higher altitudes (NFh), with forest canopy reaching the heights of up to $30 \mathrm{~m}$ and $25 \mathrm{~m}$, respectively. The vegetation cover is over $70 \%$, consisting of natural forests with some small natural gaps, characterized by the presence of trees of various families in the climax forests. The major plant species are in the families of Lauraceae, Fagaceae, Theaceae, Magnoliaceae, Ericaceae, Myrtaceae, Rubiaceae and some others.

Stream sides at the lower ( $\mathrm{SSl})$ and higher altitudes (SSh), situated along a local stream $10-15 \mathrm{~m}$ wide. The vegetation is broad leaf evergreen plants, the canopy height up to $25 \mathrm{~m}$. The main plant species are in the families Lauraceae, Fagaceae, Magnoniaceae, Theaceae, Rubiaceae, with shrubs and grasses spread along stream sides. The forest canopy along them is $30 \%$.
Grassy areas at the lower (GRl) and higher altitudes (GRh), characterized by the presence of grasses and some small shrubs. The main species are in the families Poaceae, Melastomaceae, and some others.

\section{Sampling methods}

Butterflies were counted to assess the species richness and abundance in habitats to reveal both the presence and abundance of butterfly species in different habitat types at different altitudes. The transect count method was used to record butterflies. A number of butterflies were collected on different sites beyond the transects using butterfly hand nets for identification.

The transect work was performed between 8:00 a.m. to 4:00 p.m. on fine days. To cover each 500-m transect, it took about 40-50 minutes. The sampling times for each transect were altered from day to day to reduce the effect of different times of the day on the data recorded. Each transect was repeated in 25 counts. This method was applied in some of the previous studies on the butterflies of tropical forests in Vietnam [Spitzer et al., 1997; Vu, 2007, 2009].

\section{Data analysis}

The diversity of butterfly communities and the similarity of species composition between different habitat types were calculated using Cluster Analysis [Primer, 2001]. Butterfly diversity was measured with the use of the following indices: species number, individuals, species richness index $(d)$, evenness index $J$ ', and diversity index $H^{\prime}$. The identification of butterflies follows Chou [1994], Osada et al. [1999], D’Abrera [1982-1986], Monastyrskii [2005].

\section{Results}

A total of 170 species were recorded during the study period. The species list and their abundance are presented in Table 1. There are two species listed in the Red List of Vietnam: Troides aeacus and Byasa crassipes [Ministry of Science and Technology, 2007]. The former species is also listed in CITES [New \& Collins, 1991]. Most of the species yielded 1-5 individuals in all of the habitats (112 species, $66 \%$ of all species); the species represented by more than 10 individuals are 8 (16.5\% of all species). The most abundant species is Zemeros flegyas (11.5\% of all individuals), followed by Delias belladonna $(6 \%)$.

Table 1. Species list of the butterflies and their abundance in the Van Ban Nature Reserve in March and April 2005. Таблица 1. Видовой список бабочек и их численность в заповеднике Ван-Бан в марте-апреле 2005 г.

\begin{tabular}{|c|c|c|c|c|c|c|c|}
\hline \multirow{2}{*}{ No. } & \multirow{2}{*}{ Species } & \multicolumn{3}{|c|}{ Below $700 \mathrm{~m}$} & \multicolumn{3}{|c|}{ Above $700 \mathrm{~m}$} \\
\hline & & NFl & SS1 & GRI & NFh & SSh & GRh \\
\hline \multicolumn{8}{|c|}{ Papilionidae } \\
\hline 1 & Troides aeacus (C.Felder et R.Felder, 1860) & 1 & 1 & & 1 & & \\
\hline 2 & Byasa crassipes (Oberthür, 1879) & 1 & & & 1 & & \\
\hline 3 & Atrophaneura dasarada (Moore, 1857) & 2 & & & 1 & & \\
\hline 4 & Atrophaneura varuna (White, 1868 ) & & & & & 2 & \\
\hline 5 & Chilasa clytia (Linnaeus, 1758) & & & 2 & & 2 & \\
\hline
\end{tabular}


Table 1. Continue. Таблица 1. Продолжение.

\begin{tabular}{|c|c|c|c|c|c|c|c|}
\hline \multirow{2}{*}{ No. } & \multirow{2}{*}{ Species } & \multicolumn{3}{|c|}{ Below $700 \mathrm{~m}$} & \multicolumn{3}{|c|}{ Above $700 \mathrm{~m}$} \\
\hline & & NFl & SSl & GR1 & $\mathrm{NFh}$ & SSh & GRh \\
\hline 6 & Chilasa slateri (Hewitson, 1857) & 1 & & 1 & & & 1 \\
\hline 7 & Chilasa agestor (Gray, 1831) & 2 & 2 & & 1 & 2 & \\
\hline 8 & Chilasa epycides (Hewitson, 1862) & 1 & 1 & & 4 & 3 & \\
\hline 9 & Chilasa paradoxa (Zinken, 1831) & & 1 & & & & \\
\hline 10 & Papilio helenus (Linnaeus, 1758) & 3 & 2 & 2 & 1 & 4 & 1 \\
\hline 11 & Papilio nephelus (Boisduval, 1836) & & 2 & & & & \\
\hline 12 & Papilio polytes (Linnaeus, 1758) & & 2 & 2 & & 2 & 1 \\
\hline 13 & Papilio memnon Linnaeus, 1758 & 2 & & 1 & 1 & 1 & 1 \\
\hline 14 & Papilio paris Linnaeus, 1758 & 3 & 2 & 4 & & 1 & 1 \\
\hline 15 & Papilio bianor (Cramer, 1777) & 1 & 2 & 1 & & 2 & \\
\hline 16 & Papilio protenor Cramer, 1775 & 2 & 2 & & 1 & 2 & 1 \\
\hline 17 & Papilio bootes nigricans (Rothschild, 1895) & & 14 & & & 2 & \\
\hline 18 & Meandrusa sciron Leech, 1890 & & & & 1 & & \\
\hline 19 & Meandrusa payeni (Boisduval, 1836) & 1 & & & 1 & & \\
\hline 20 & Graphium antiphates (Cramer, 1775) & & & & 1 & & 1 \\
\hline 21 & Graphium doson (C.Felder et R.Felder, 1864) & & 3 & & & & \\
\hline 22 & Graphium xenocles (Doubleday, 1842) & 1 & 2 & & 2 & 2 & 2 \\
\hline 23 & Graphium megarus (Westwood, 1844) & & 1 & & & & \\
\hline 24 & Graphium macareus (Godart, 1819) & & 1 & & & & \\
\hline 25 & Graphium chironides (Honrath, 1884) & & 3 & & & & \\
\hline 26 & Graphium agamemnon (Linnaeus, 1758) & & 1 & 2 & & 1 & 1 \\
\hline 27 & Graphium sarpedon (Linnaeus, 1758) & 2 & 2 & 1 & 1 & 2 & 1 \\
\hline 28 & Graphium agetes (Westwood, 1843) & & 6 & & & & \\
\hline 29 & Graphium cloanthus (Westwood, 1841) & & 1 & & & & \\
\hline 30 & Graphium eurous (Leech, 1893) & & 6 & & & 4 & \\
\hline 31 & Lamprotera meges (Zinken-Sommet, 1831) & & & & & 1 & \\
\hline \multicolumn{8}{|c|}{ Pieridae } \\
\hline 32 & Delias pasithoe (Linnaeus, 1767) & 1 & 1 & & 1 & & \\
\hline 33 & Delias hyparete (Linnaeus, 1758) & 1 & 1 & & 2 & & \\
\hline 34 & Delias belladonna (Fabricius, 1793) & 8 & 30 & & 2 & 35 & \\
\hline 35 & Prioneris thestylis (Doubleday, 1842) & & 2 & 2 & & 2 & 1 \\
\hline 36 & Artogeia napi (Linnaeus, 1758) & & & 1 & & & \\
\hline 37 & Pieris canidia (Linnaeus, 1768) & & 2 & 9 & & & 7 \\
\hline 38 & Talbotia naganum (Moore, 1884) & & 2 & & & & \\
\hline 39 & Hebomoia glaucippe (Linnaeus, 1758) & 1 & 1 & 1 & 1 & & 1 \\
\hline 40 & Appias albina (Boisduval, 1836) & 1 & & 1 & & & 1 \\
\hline 41 & Appias lyncida (Cramer, 1777) & & & 1 & & & \\
\hline 42 & Appias indra (Moore, 1857) & 1 & 1 & & & & \\
\hline 43 & Appias lalage (Doubleday, 1842) & & 1 & & & 1 & \\
\hline 44 & Dercas verhuelli (van de Hoeven, 1839) & 1 & 1 & & 2 & 1 & \\
\hline 45 & Catopsilia pomona (Fabricius, 1775) & 6 & 2 & & & 4 & 3 \\
\hline 46 & Eurema andersonii (Moore, 1886) & & 1 & 1 & & & 1 \\
\hline \multicolumn{8}{|c|}{ Danaidae } \\
\hline 47 & Parantica sita (Kollar, 1844) & & & & 4 & 5 & 1 \\
\hline 48 & Parantica melaneus (Cramer, 1775) & 2 & 1 & 2 & 3 & 2 & 1 \\
\hline 49 & Parantica aglea (Stoll, 1782) & 1 & & 1 & & & \\
\hline 50 & Ideopsis similis (Linnaeus, 1758) & & & 1 & & & \\
\hline 51 & Euploea mulciber (Cramer, 1777) & 2 & 1 & 3 & 1 & 1 & 1 \\
\hline
\end{tabular}


Table 1. Continue. Таблица 1. Продолжение.

\begin{tabular}{|c|c|c|c|c|c|c|c|}
\hline \multirow{2}{*}{ No. } & \multirow{2}{*}{ Species } & \multicolumn{3}{|c|}{ Below $700 \mathrm{~m}$} & \multicolumn{3}{|c|}{ Above $700 \mathrm{~m}$} \\
\hline & & NFl & SSl & GR1 & $\mathrm{NFh}$ & SSh & GRh \\
\hline \multicolumn{8}{|c|}{ Satyridae } \\
\hline 52 & Melanitis leda (Linnaeus, 1758) & 1 & 1 & & 1 & & \\
\hline 53 & Elymnias hypermnestra (Linnaeus, 1763) & & & 1 & & & \\
\hline 54 & Lethe siderea Marshall, 1880 & & & & 2 & & \\
\hline 55 & Lethe dura (Marshall, 1882) & & & & 2 & 1 & \\
\hline 56 & Lethe confusa Aurivillius, 1897 & 1 & 1 & 2 & & 1 & \\
\hline 57 & Lethe verma (Kollar, 1844) & 1 & 1 & & 1 & & 1 \\
\hline 58 & Lethe chandica (Moore, 1858) & & & & 2 & & \\
\hline 59 & Lethe syrcis (Hewitson, 1863) & 2 & 1 & & 1 & 2 & \\
\hline 60 & Lethe insana (Kollar, 1844) & 1 & 1 & & 2 & & \\
\hline 61 & Lethe vyndhia (C.Felder et R.Felder, 1859) & 1 & & & 3 & 1 & \\
\hline 62 & Lethe mekara (Moore, 1858) & & & & 2 & & \\
\hline 63 & Lethe sinorix (Hewitson, 1863) & 2 & & & & & \\
\hline 64 & Lethe kansa (Moore, 1857) & 1 & & & 1 & & \\
\hline 65 & Lethe naga Doherty, 1889 & 6 & 4 & & 7 & & \\
\hline 66 & Lethe gulnihal de Nicéville, 1887 & & & & 3 & & \\
\hline 67 & Lethe berdievi Monastyrskii, 2005 & & & & 2 & & \\
\hline 68 & Neope muirheadi (C.Felder et R.Felder, 1862) & 6 & 1 & & 7 & 1 & \\
\hline 69 & Neope bhadra (Moore, 1857) & 4 & & & 3 & & \\
\hline 70 & Neope pulaha (Moore, 1858) & & & & 1 & & \\
\hline 71 & Mycalesis anaxias aemate Fruhstorfer, 1911 & 5 & & & 6 & & \\
\hline 72 & Mycalesis adamsonii Watson, 1897 & 2 & & & & & \\
\hline 73 & Mycalesis misenus de Nicéville, 1901 & 6 & & 1 & 8 & & \\
\hline 74 & Mycalesis francisca (Stoll, 1780) & 3 & 1 & 3 & 7 & & 2 \\
\hline 75 & Ragadia crisilda Hewitson, 1862 & 1 & & & 2 & & \\
\hline 76 & Ypthima baldus (Fabricius, 1775) & & & 7 & & & 7 \\
\hline 77 & Ypthima frontier Uémura et Monastyrskii, 2000 & & & & & & 3 \\
\hline 78 & Callerebia narasingha (Moore, 1857) & & 15 & & & 2 & \\
\hline \multicolumn{8}{|c|}{ Amathusiidae } \\
\hline 79 & Aemona amathusia (Hewitson, 1867) & 2 & & & 3 & & \\
\hline 80 & Enispe euthymius (Doubleday, 1845) & 2 & & & 1 & & \\
\hline 81 & Discophora sondaica Boisduval, 1836 & 1 & 1 & & & & \\
\hline \multicolumn{8}{|c|}{ Nymphalidae } \\
\hline 82 & Ariadne merione (Cramer, 1777) & & 3 & & & & 2 \\
\hline 83 & Argyreus hyperbius (Linnaeus, 1763) & & & 6 & & & 5 \\
\hline 84 & Vagrans egista (Cramer, 1780) & & & 2 & & & 1 \\
\hline 85 & Vanessa cardui (Linnaeus, 1758) & 1 & & & & & \\
\hline 86 & Symbrenthia lilaea (Hewitson, 1864) & & & 1 & & & \\
\hline 87 & Lasippa tiga (Moore, 1858) & & 2 & & & 2 & 2 \\
\hline 88 & Pantoporia hordonia (Stoll, 1790) & & 1 & & & 1 & \\
\hline 89 & Hypolimnas bolina (Linnaeus, 1758) & 1 & 1 & & & & \\
\hline 90 & Kallima inachus (Boisduval, 1846) & 2 & 1 & & 3 & & 1 \\
\hline 91 & Cyrestis thyodamas Boisduval, 1836 & & 2 & & & 2 & \\
\hline 92 & Chersonesia risa (Doubleday, 1848) & 1 & & & 1 & 1 & \\
\hline 93 & Neptis clinia (Moore, 1872) & & 2 & & & 2 & \\
\hline 94 & Neptis miah Moore, 1858 & & 1 & & & & \\
\hline 95 & Neptis ananta Moore, 1858 & & 2 & & & 2 & \\
\hline 96 & Neptis namba Tytler, 1915 & & 4 & & & 5 & 3 \\
\hline 97 & Neptis yerburii Butler, 1886 & & 2 & & 2 & 1 & 3 \\
\hline
\end{tabular}


Table 1. Continue. Таблица 1. Продолжение.

\begin{tabular}{|c|c|c|c|c|c|c|c|}
\hline \multirow{2}{*}{ No. } & \multirow{2}{*}{ Species } & \multicolumn{3}{|c|}{ Below $700 \mathrm{~m}$} & \multicolumn{3}{|c|}{ Above $700 \mathrm{~m}$} \\
\hline & & NFI & SSl & GR1 & $\mathrm{NFh}$ & SSh & GRh \\
\hline 98 & Neptis harita Moore, 1875 & & 2 & & & 2 & \\
\hline 99 & Athyma selenophora (Kollar, 1844) & & 1 & & & 1 & \\
\hline 100 & Athyma ranga Moore, 1857 & & & & & 1 & 1 \\
\hline 101 & Athyma cama Moore, 1858 & & 2 & & & 2 & \\
\hline 102 & Athyma nefte (Cramer, 1780) & & 2 & & & & \\
\hline 103 & Athyma asura Moore, 1858 & & 1 & & & 1 & \\
\hline 104 & Athyma zeroca Moore, 18725 & & 3 & & & 2 & \\
\hline 105 & Athyma pravara Moore, 1857 & & & & & 1 & \\
\hline 106 & Sumalia daraxa (Doubleday, 1848) & & & & 2 & 2 & 2 \\
\hline 107 & Moduza procris (Cramer, 1777) & 1 & 1 & & & 2 & \\
\hline 108 & Tanaecia julii (Lesson, 1837) & & 1 & & & & \\
\hline 109 & Euthalia franciae (Gray, 1846) & & & & 1 & & \\
\hline 110 & Lexias dirtea (Fabricius, 1793) & 2 & & & 1 & 2 & \\
\hline 111 & Auzakia danava (Moore, 1858) & 2 & & & 1 & & \\
\hline 112 & Eulacera osteria (Westwood, 1850) & 2 & & & & & \\
\hline 113 & Hestina nama (Doubleday, 1844) & & & & & & 2 \\
\hline 114 & Stibochiona nicea (Gray, 1846) & 2 & & & & & \\
\hline 115 & Dichorragia nesimachus (Doyere, 1840) & 1 & & & & & \\
\hline 116 & Charaxes bernardus (Fabricius, 1793) & & 1 & & & & \\
\hline 117 & Charaxes aristogiton C.Felder, 1867 & & & & 2 & & \\
\hline 118 & Polyura athamas (Drury, 1773) & & 2 & & 1 & 2 & \\
\hline 119 & Polyura nepenthes (Grose-Smith, 1883) & & 4 & & & & \\
\hline 120 & Polyura narcaea (Hewitson, 1854) & & 15 & & & 2 & 1 \\
\hline 121 & Polyura dolon (Westwood, 1848) & & 15 & & & 4 & \\
\hline 122 & Polyura eudamippus (Doubleday, 1843) & & 12 & & & 6 & \\
\hline 123 & Calinaga buddha (Moore, 1857) & & 2 & 1 & & & \\
\hline & Riodinidae & & & & & & \\
\hline 124 & Abisara fylla (Westwood, 1851) & 7 & 6 & & 8 & 6 & \\
\hline 125 & Abisara neophron (Hewitson, 1861) & & & & 3 & & \\
\hline 126 & Abisara burnii (de Nicéville, 1895) & & 1 & & 1 & & \\
\hline 127 & Zemeros flegyas (Cramer, 1843) & 18 & 30 & 45 & 10 & 22 & 35 \\
\hline 128 & Stiboges nymphidia (Butler, 1876) & 2 & & & 2 & & \\
\hline 129 & Dodona ouida (Hewitson, 1865) & 6 & & & 2 & & \\
\hline 130 & Dodona egeon (Westwood, 1851) & & & 1 & & 2 & \\
\hline 131 & Dodona dipoea (Hewitson, 1865) & & 1 & & & 1 & \\
\hline 132 & Dodona adonira (Hewitson, 1865) & & & & 1 & & \\
\hline & Lycaenidae & & & & & & \\
\hline 133 & Miletus chinensis C.Felder, 1862 & 3 & & & 2 & & \\
\hline 134 & Neopithecops corvus Fruhstorfer, 1919 & & 1 & & & & \\
\hline 135 & Neopithecops zalmora (Butler, 1870) & & 1 & & & & \\
\hline 136 & Acytolepis puspa (Horsfield, 1828) & & 3 & & & 2 & \\
\hline 137 & Celastrina argiolus (Linnaeus, 1758) & 2 & 12 & & 1 & 2 & \\
\hline 138 & Celastrina lavendularis (Moore, 1877) & 3 & 4 & & & & \\
\hline 139 & Zizeeria maha (Kollar, 1844) & & & 3 & & & 2 \\
\hline 140 & Udara dilecta (Moore, 1879) & & 7 & & & 2 & \\
\hline 141 & Jamides alecto (C.Felder, 1860) & & & & & 1 & \\
\hline 142 & Jamipes bochus (Stoll, 1782) & & 2 & & & & \\
\hline 143 & Ancema ctesia (Hewitson, 1865) & & 1 & & & & \\
\hline 144 & Heliophorus ila (de Nicéville et Martin, 1896) & & & 1 & & 1 & 1 \\
\hline
\end{tabular}


Table 1. Continue. Таблица 1. Продолжение.

\begin{tabular}{|c|c|c|c|c|c|c|c|}
\hline \multirow{2}{*}{ No. } & \multirow{2}{*}{ Species } & \multicolumn{3}{|c|}{ Below $700 \mathrm{~m}$} & \multicolumn{3}{|c|}{ Above $700 \mathrm{~m}$} \\
\hline & & $\mathrm{NF1}$ & SS1 & GRI & NFh & SSh & GRh \\
\hline 145 & Surendra quercetorum (Moore, 1857) & 2 & 3 & & 2 & 1 & \\
\hline 146 & Tajuria sp. (?maculata Moore, 1883) & 1 & & & & & \\
\hline 147 & Tajuria diaeus (Hewitson, 1865) & 1 & & & 1 & & \\
\hline 148 & Chliaria kina kina (Hewitson, 1869) & 1 & & & 1 & 2 & \\
\hline 149 & Curetis bulis Westwood, 1851 & & 12 & 1 & & 6 & \\
\hline & Hesperiidae & & & & & & \\
\hline 150 & Choaspes benjaminii (Guérin-Méneville, 1843) & & & & & 1 & \\
\hline 151 & Celaenorrhinus inaequalis Elwes et Edwards, 1897 & & & & & 1 & \\
\hline 152 & Celaenorrhinus vietnamicus Devyatkin, 1998 & & 1 & & & & \\
\hline 153 & Tagiades menaka (Moore, 1865) & & 2 & 1 & 3 & 1 & \\
\hline 154 & Tagiades parra Fruhstorfer, 1910 & & & 1 & & & \\
\hline 155 & Seseria dohertyi Watson, 1893 & & 1 & & & & \\
\hline 156 & Gerosis phisara Moore, 1884 & 1 & & & & & \\
\hline 157 & Mooreana trichoneura (C.Felder et R. Felder, 1860) & 1 & & & & & \\
\hline 158 & Odontoptilum angulata (C.Felder, 1862) & & 2 & & & & \\
\hline 159 & Thoressa gupta de Nicéville, 1886 & & 7 & & & 13 & \\
\hline 160 & Halpe frontieri Devyatkin, 1997 & & 2 & & 6 & 3 & 1 \\
\hline 161 & Iambrix salsala (Moore, 1865) & 1 & 1 & 1 & 1 & 1 & \\
\hline 162 & Koruthaialos sindu C.Felder et R. Felder, 1860 & & 1 & & & & \\
\hline 163 & Notocrypta curvifascia (C.Felder et R. Felder, 1860) & 2 & 2 & & & & 1 \\
\hline 164 & Potanthus mingo (Edwards, 1866) & 1 & & 2 & & 1 & \\
\hline 165 & Ochlodes siva Moore, 1878 & & 12 & & & 15 & \\
\hline 166 & Pithauria stramineipennis Wood-Mason et de Nicéville, 1868 & 2 & 10 & 3 & & & \\
\hline 167 & Satarupa gopala Moore, 1865 & & & & 1 & & \\
\hline 168 & Ampitia dioscorides (Fabricius, 1793) & & 1 & & & & \\
\hline 169 & Barba bicolor (Oberthür, 1896) & & 1 & & & & \\
\hline 170 & Creteus cyrina (Hewitson, 1876) & & & & & 1 & \\
\hline
\end{tabular}

NOTE: NF1/NFh — Natural forest at low/high altitude; SBl/SSh — stream sides in forest at low/high altitude; GR1/GRh — grassy areas at low/high altitude.

ПРИМЕЧАНИЕ: NF1/NFh - естественные леса в низко/высокогорье; SB1/SSh — берега рек в низко/высокогорье; GR1/ $\mathrm{GRh}$ - луга в низко/высокогорье.

The diversity of butterfly communities per habitat is presented in Table 2. Stream sides appear to support the greatest number of species and individuals: 97 species and 342 individuals, and 68 species and 223 individuals at the lower and higher altitudes, respectively. The most abundant species along the stream sides are D. belladonna, Z. flegyas, Ochlodes siva, Thoressa gupta, Callerebia narasingha, Polyura narcaea, P. dolon, P. eudamippus, Celastrina argiolus, Curetis bulis, and Papilio bootes. They make $50 \%$ of all individuals recorded in stream side habitats at both altitudes compared.

Grassy habitats show the least species and individual numbers: 39 species and 122 individuals, and 39 species and 104 individuals at the lower and higher altitudes, respectively. The most abundant species in these habitats are Z. flegyas, Pieris canidia, Ypthima baldus, and Argyreus hyperbius. They make 53\% of all individuals recorded in the grassy habitats at both altitudes compared.

Natural forests yield more species and individual numbers than the grass habitats: 71 species and 165 individuals, and 68 species and 159 individuals at the lower and higher altitudes, respectively. The most abundant species of the natural forest habitats are Z. flegyas, Mycalesis misenus, M. anaxias, Lethe naga and Neope muirrheadi. They compose $46 \%$ of all individuals recorded in the natural forest habitats at both altitudes compared.

The abundance index of butterfly communities is the highest along stream sides at both altitudes: 16.45 and 13.32, respectively. Grassy habitats have the least abundance index. The highest evenness index of butterfly communities is in natural forests, while the lowest evenness indices are in grassy habitats. The diversity indices of butterfly communities both along stream sides and in natural forests are similar: 3.91 and 3.93 in natural forest at the lower and higher altitudes, versus 3.95 and 3.68 along stream sides at the lower and higher altitudes, respectively.

The habitats at the lower elevations yield more species and individuals than those at the higher altitude, 
Table 2. Butterfly diversity in different habitat types at Van Ban.

Таблица 2. Разнообразие дневных бабочек в разных типах биотопов в Ван-Бан.

\begin{tabular}{|c|c|c|c|c|c|}
\hline 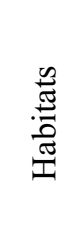 & 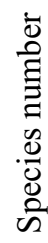 & 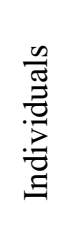 & 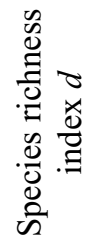 & 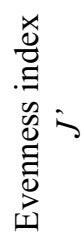 & 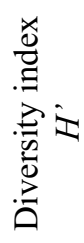 \\
\hline NFl & 71 & 165 & 13.71 & 0.92 & 3.91 \\
\hline $\mathrm{SSl}$ & 97 & 342 & 16.45 & 0.86 & 3.95 \\
\hline GRl & 39 & 122 & 7.91 & 0.76 & 2.78 \\
\hline NFh & 68 & 159 & 13.22 & 0.93 & 3.93 \\
\hline SSh & 73 & 223 & 13.32 & 0.86 & 3.68 \\
\hline GRh & 39 & 104 & 8.19 & 0.79 & 2.89 \\
\hline
\end{tabular}

NOTE: habitats as in Table 1.

ПРИМЕЧАНИЕ: обозначения биотопов как в Табл. 1.

with the exception of species in grassy habitats: 71 and 68 species in natural forests at the lower and higher altitudes, and 97 and 73 species along stream sides at the lower and higher elevations, respectively. The number of species recorded in all habitats at the lower altitude is higher than that revealed in all habitats at the higher elevation, totaling 144 and 126 species, respectively. The abundance levels show the same trend: 629 and 486 individuals, respectively.

The similarity of species compositions between different habitats and altitudes is presented in Figure. The species composition appears to be similar in similar habitats: stream sides at both altitudes, natural forests at both altitudes, and grassy habitats at both altitudes.

\section{Discussion}

Natural tropical forests support less butterfly species and individuals than stream sides, being darker, with thick canopies and less gaps. In addition, the areas under the forest canopy are almost devoid of flowering plants which support numerous butterflies. Warren [1985] emphasized fewer butterfly species occurring in habitats with thick forest canopies, as opposed to more numerous butterfly species found in the habitats with less dense forest canopies. Indeed, natural tropical forests fail to show high levels of species diversity, but most of the species living there are forest-dwellers with small geographical distributions [Spitzer et al., 1997; Vu, 2007, 2009]. Thus, natural forests at Van Ban contain many species in the mainly sylvicolous families Satyridae and Amathusiidae: 27 and 30 species, respectively.

Stream sides in the forest yield more species and individuals of butterflies than both natural forest and the grassy areas. The environment along stream sides is especially diversified with vegetation, rocks, sand, mud and water which all attract more butterflies as they land for taking water and nutrients. Generally, wet areas are long known to attract more butterflies than dry sites do [Janzen \& Schoener, 1968]. In addition, stream sides in the forest have openings which support more butterflies. Gaps in the forest have higher butterfly diversity levels than closed forest does [Spitzer et al., 1997]. Along streams, shrub and grass with flowering plants also sustain more butterflies. Expectedly, stream sides show fewer species from the basically sylvicolous families Satyridae and Amthusiidae: 12 and 30 species, respectively.

Grassy habitats support less butterfly species and individuals than both natural forests and stream sides, because they are very simple, with most of the grasses
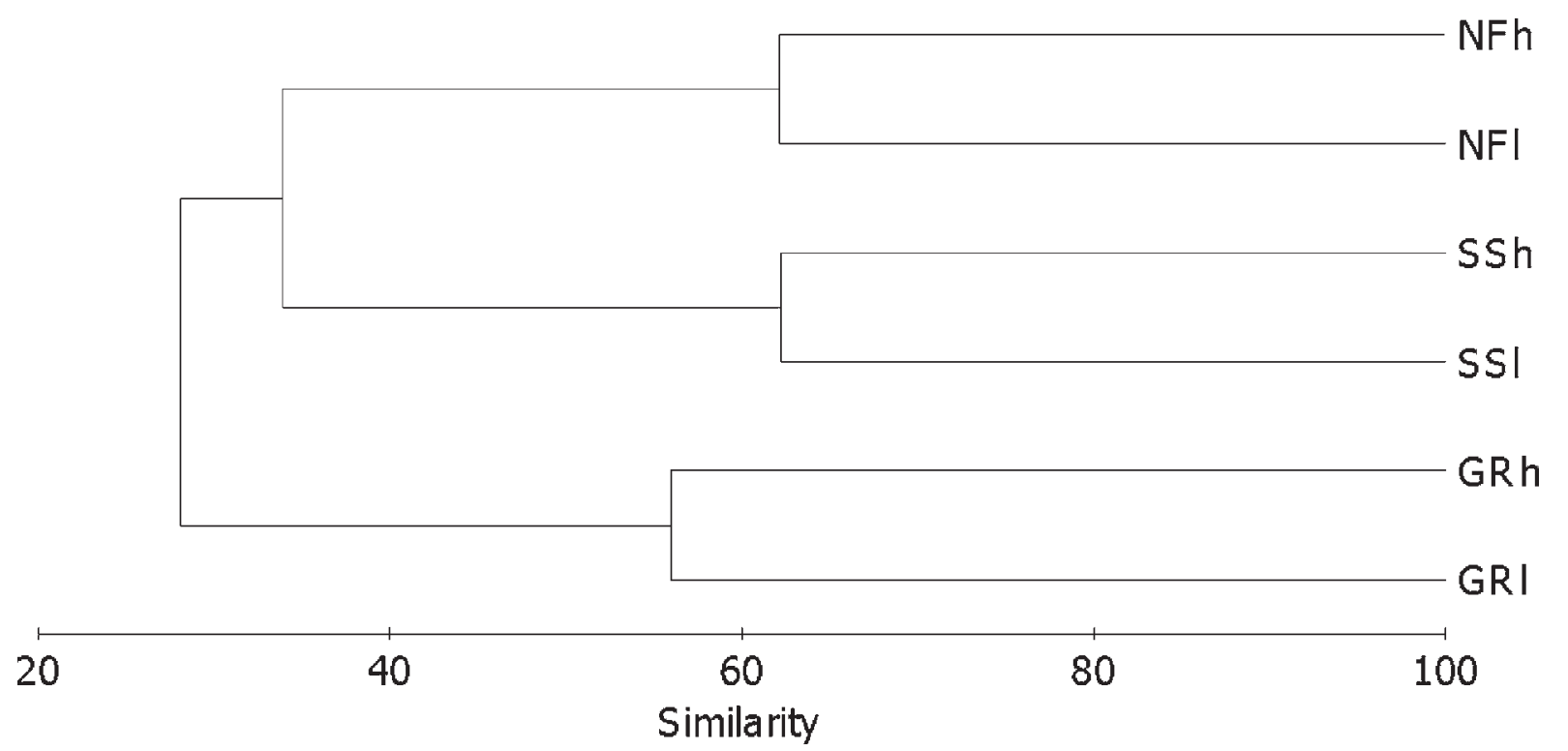

Figure. Similarity of butterfly species composition in different habitat types.

Рисунок. Сходство видового состава бабочек в разных типах биотопов. 
giving home to only few butterflies. Unsurprisingly, there are very few species from the families Satyridae and Amthusiidae living in grassy habitats at Van Ban: 8 and 30 species, respectively. The lower diversity of vegetation leads to lower diversity levels of butterflies: as other studies show, the greater the diversity of plants, the higher the diversity of butterflies and other insects [Spitzer et al., 1987; DeVries, 1992]. These results correspond well to our previous work as well [ $\mathrm{Vu} 2009$; Vu \& Yuan, 2003], emphasizing that shrub and grassy habitats have the species and abundance levels of butterfly communities lower than those in natural forests.

Lower altitudes appear to harbour more species and individuals of butterflies than higher ones do. Such results correspond well to the theory and practice demonstrated in previous work, indicating that the diversity of insects or butterflies decreases with an increasing latitude or altitude [Sparrow et al., 1994; Vu and Yuan, 2003].

Species composition was dissimilar among all habitats, but rather similar between similar habitats: natural forests at both altitudes, stream sides at both altitudes, and grassy areas at both altitudes. This proves that habitat is the main factor to affect the similarity or dissimilarity of butterfly communities between habitats [Vu, 2008; Vu et al., 2008]. The butterfly species composition differed between habitat types. This result is similar to those obtained by Steffan-Dewenter \& Tscharntke [1997] and Vu [2009].

The results of the present study support the hypothesis that the environment of forest stream sides supports particularly high diversity levels of butterfly species and abundance, as opposed to those observed in grassy habitats. Thus, stream sides are highly important environments for butterfly conservation.

ACKNOWLEDGEMENTS. I am most grateful to S. Golovatch (Moscow, Russia) who kindly edited the English of an advanced draft. Thanks are also due to the VietnamRussian Tropical Center which supported the research.

\section{References}

Barlow H.S. \& Woiwod I.P. 1989. Moth diversity of a tropical forest in Peninsular Malaysia // J. Trop. Ecol. Vol.5. P.37-50.

Blair R.B. \& Launer A.E. 1997. Butterfly diversity and human land use: species assemblages along an urban gradient // Biological Conservation. Vol.80. P.113-125.

Bobo K.S., Waltert M., Fermon H., Njokagbor J. \& Muhlenberg M. 2006. From forest to farmland: butterfly diversity and habitat associations along a gradient of forest conversion in southwestern Cameroon // Journal of Insect Conservation. Vol.10. P.29-42.

Brown K.S. 1991. The conservation of insects and their habitats // Conservation of Neotropical environments: Insects as indicators, 15th Symposium of the Royal Entomological Society of London, September 1989. Academic Press, London, England. P.350-403.

Chou L. 1994. Monographia Rhopalocerum Sinensium. Vols 1-2. Henan Science and Technology Press, China.

D'Abrera B. 1982-86. Butterflies of the Oriental Region. Vol.1-3. Hill House, Melbourne.
DeVries R.G. 1992. Outlines of Entomology. $7^{\text {th }}$ edition. Chapman \& Hall

Fermon H., Waltert M., Vane-Wright R.I. \& Muhlenberg M. 2005. Forest use and vertical stratification in fruit-feeding butterflies of Sulawesi, Indonesia: impacts for conservation // Biodiversity and Conservation. Vol.14. P.333-350.

Hill J.K., Hamer K.C., Dawood M.M., Tangah J. \& Dawood D. 2001. Ecology of tropical butterflies in rainforest gaps // Oecologia, Vol.128. P.294-302.

Janzen D.H. \& Schoener T.W. 1968. Difference in insect abundance and diversity between wetter and drier sites during a tropical dry season // Ecology. Vol.49. P.96-110.

Ministry of Science and Technology. 2007. Vietnam Red List. The Science and Techonology Publishing House, Hanoi [in Vietnamese].

Monastyrskii A.L. 2005. Butterflies of Vietnam, Nymphalidae: Satyrinae. Vol.1. Cartographic Publishing House, Hanoi, Vietnam.

Morse D.R., Stork N.E. \& Lawton J.H. 1988. Species number, species richness and body length relationships of arboreal beetles in Bornean low land rain forest trees // Ecological Entomology. Vol.13. P.25-37.

New T.R. \& Collins N.M. 1991. Swallowtail Butterflies: An action plan for their conservation. IUCN, Gland, Switzerland.

Osada S., Uemura Y. \& Uehara J. 1999. An illustrated checklist of the butterflies of Laos P.D.R. Tokyo, Japan

Primer-E Ltd. 2001. Primer 5 for Windows. Version 5.2.4.

Schulze C.H., Steffan-Dewenter I. \& Tsharntke T. 2004. Effect of land use on butterfly communities at the rainforest margin: a case study from Central Sulawesi // G. Gerold , M. Fremerey \& E. Guhardja (Eds). Land Use, Nature Conservation and the Stability of Rainforest Margins in Southeast Asia. SpringerVerlag Berlin \& Heidelberg. P.281-297.

Sparrow R.H., Sisk D.T., Ehrlich P.R. \& Murphy D.D. 1994. Techniques and Guidelines for monitoring Neotropical butterflies // Conservation Biology. Vol.8. P.800-809.

Spitzer K., Jaroś J., Havelka J. \& Lepš J. 1997. Effect of small-scale disturbance on butterfly communities of an Indochina montane rainforest // Biological Conservation. Vol.80. P.9-15.

Spitzer K., Lepš J. \& Soldan T. 1987. Butterfly communities and habitat of seminatural savana in southern Vietnam (Papilionoidae, Lepidoptera) // Acta Entomol. Bohemoslov. Vol.84. P.200-208.

Spitzer K., Novotny V., Tonner M. \& Lepš J. 1993. Habitat preferences, distribution and seasonality of the butterflies (Lepidoptera, Papilionoidae) in a montane tropical rain forest, Vietnam // Journal of Biogeography. Vol.20. P.109-121.

Steffen-Dewenter I. \& Tscharntke T. 1997. Early succession of butterfly and plant communities on set-aside fields // Oecologia. Vol.109. P.294-302.

Vu V.L. 2007. Ecological indicator role of butterflies in Tam Dao National Park, Vietnam // Russ. Entomol. J. Vol.16. P.473-480.

Vu V.L. 2008. Biodiversity of butterflies (Lepidoptera: Rhopalocera) and ecological indicators of butterfly species in Tam Dao National Park, Vinh Phuc. PhD Thesis, Institute of Ecology and Biological Resources, Hanoi, Vietnam [in Vietnamese].

Vu V.L. 2009. Diversity and similarity of butterfly communtities in five different habitat types at Tam Dao National Park, Vietnam // Journal of Zoology. Vol.27. P.15-22.

Vu V.L., Vu Q.C. \& Ta H.T. 2008. The study result of butterflies (Lep.: Rhopalocera) composition and their distribution in different habitats and altitudes in Tam Dao National Park // Proceedings of the $6^{\text {th }}$ Vietnam National Conference on Entomology. Agricultural Publishing House, Hanoi. P.188-203 [in Vietnamese].

Vu V.L. \& Yuan D.C. 2003. The differences of butterfly (Lepidoptera, Papilionoidea) communities in habitats with various degrees of disturbance and altitudes in tropical forests of Vietnam // Biodiversity and Conservation. Vol.12. P.1099-1111.

Warren M.S. 1985. The influence of shade on butterfly numbers in woodland rides, with special reference to the Wood White Leptidea sinapis // Biological Conservation. Vol.33. P.147-164. 